\title{
STELLAR ENVELOPES AT SUPERCRITICAL LUMINOSITY
}

\section{(Abstract)}

\author{
G. S. BISNOVATYI-KOG AN
}

Institute of Applied Mathematics, USSR Academy of Sciences, Moscow, U.S.S.R.

Analytical solutions are obtained for static envelopes in radiative equilibrium for a dependence of opacity $\varkappa$ on temperature $T$ given by

$$
\begin{aligned}
& \varkappa=\varkappa_{0}, \quad T>T_{1}, \quad L / L_{c 0}<1 \\
& \varkappa=\varkappa_{1}\left(T / T_{1}\right)^{n}, \quad T<T_{1}, \quad L / L_{c 1}>1,
\end{aligned}
$$

where $L_{c 0}$ and $L_{c 1}$ are critical luminosities corresponding to $\varkappa_{0}$ and $\varkappa_{1}$.

It is shown that there is an exact boundary between the regions of existence of static radiative envelopes and stationary outflowing envelopes, for which numerical solutions were obtained.

It is shown that convection makes the region of static envelopes broader and leads to the existence, for the same parameters, of both outflowing and static convective envelopes; i.e. to double-valued solutions. Such lack of uniqueness may be met during the computation of late stages of stellar evolution. 